\title{
Conformation of the Propagating Radical of Triphenylmethyl Methacrylate as Studied by ESR
}

\author{
Mikiharu KamachI, Yoko KUWAE, and Shun-ichi NozaKURA \\ Department of Macromolecular Science, Faculty of Science, \\ Osaka University, Toyonaka, Osaka 560, Japan. \\ Koichi HATADA and Heimei YUKI \\ Department of Chemistry, Faculty of Engineering Science, \\ Osaka University, Toyonaka, Osaka 560, Japan.
}

(Received October 7, 1980)

\begin{abstract}
An ESR study on the radical polymerization of triphenylmethyl methacrylate was carried out in 2-methyltetrahydrofuran over a temperature range from $-90^{\circ} \mathrm{C}$ to $30^{\circ} \mathrm{C}$. A wellresolved 9-line spectrum was observed at $-90^{\circ} \mathrm{C}$, a 5-line spectrum at $-50^{\circ} \mathrm{C}$, and a 13 -line spectrum at $0^{\circ} \mathrm{C}$. When the observation temperature was raised from $0^{\circ} \mathrm{C}$ to $30^{\circ} \mathrm{C}$, a 5 -line spectrum was recorded again. This change in the spectrum with temperature was explained on the basis of two stable conformations and the interchange between them through the free rotation of the radical end. The existence of these two conformations was supported by the calculation of conformation energy.
\end{abstract}
KEY WORDS ESR / Propagating Radical / Radical Polymerization / Triphenylmethyl Methacrylate / Conformation / Interchange / Free Rotation / $\alpha$-Methylbenzyl Methacrylate / Glassy State /

It was previously reported that the solvent effect on the $k$ p could be explained in terms of the donoracceptor complex of the propagating radicals with aromatic solvents. ${ }^{1-5}$ The ESR study of the polymerization of methyl methacrylate (MMA) and its homologs in the solvents was undertaken in an effort to confirm the presence of the complex. A number of papers have been published on the ESR study of the poly(methyl methacrylate) radical, which gives $9(5+4)$-line, $13(5+8)$-line or 5 -line spectrum according to the measurement conditions. However, the origin of these spectra is still not completely elucidated.

We previously reported an ESR study on the propagating radical of methyl methacrylate (MMA), deuterated-MMA $\left(\alpha-\mathrm{CD}_{3}\right.$ or $\left.\beta-d_{2}\right)$ and triphenylmethyl methacrylate (TPMA) in aromatic rigid glass, and discussed the origin of the $9(5+4)$ line spectrum of the poly(MMA) radical. ${ }^{6}$ When the ESR spectrum of the poly(MMA) radical was measured in 2-methyltetrahydrofuran (MTHF) rigid glass, a well-resolved $13(5+8)$-line spectrum was observed at $-150^{\circ} \mathrm{C}$ instead of $9(5+4)$-line spectrum, suggesting that the properties of the glassy matrix influenced the conformation of the propagating radical. ${ }^{6}$ In order to confirm this a more detailed ESR study on the poly(MMA) radical was attempted in MTHF. No ESR spectrum, however, was observed above $-130^{\circ} \mathrm{C}$ in the polymerization system, since the poly(MMA) radical is too unstable to be observed in a fluid medium. Instead, we found that the ESR spectrum of the poly(triphenylmethyl methacrylate) [poly(TPMA)] radical with a bulky side group could be observed in MTHF even at room temperature. Temperature dependence of the ESR spectrum of the poly(TPMA) radical was investigated over a wide temperature region in MTHF; this spectrum was explained on the basis of two conformations and the interchange between them through free rotation of the radical end. The existence of the two conformations was supported by the calculation of conformation energy. 


\section{EXPERIMENTAL}

ESR spectra were recorded on a Japan Electron Optics Laboratory Model JES-FE 1X ESR spectrometer with a $100 \mathrm{kHz}$ modulator. An ES-VT-3A variable temperature accessory was employed at a temperature other than the ambient temperature; the temperature of each sample was measured by a thermocouple placed in the cavity. Specimens for ESR study were prepared according to the following procedure: about $1 \mathrm{ml}$ of a solution of monomer $(2 \mathrm{M})$ and di-tert-butyl peroxide (DTBP $0.1 \mathrm{M})$ in MTHF was put into a spectrocil sample tube $(2 \mathrm{~mm}$ diameter), and degassed by repeated melting and freezing under vacuum, and then sealed. The ESR spectra were measured under irradiation through a slotted opening of the cavity with a 500 watt high pressure mercury lamp (Ushio 500) at various temperatures.

TPMA was prepared from silver methacrylate and triphenylmethylchloride in diethyl ether. ${ }^{7} \alpha$ Methylbenzyl methacrylate was prepared from methacryloyl chloride and the corresponding carbinol. ${ }^{7}$ Di-tert-butyl peroxide was purified by reduced pressure distillation at a low temperature. MTHF was dried with calcium dihydride, which was then filtered off, and distilled over sodium.

\section{CALCULATION}

The conformation energy calculation was carried out taking into account the internal rotation, the nonbonded atomic interaction and the electrostatic interaction. ${ }^{8}$ The bond length, the bond angle, and other parameters for the calculation were taken from the work of Tadokoro et al. on isotactic poly(MMA). ${ }^{9}$ The carbon atom having an unpaired electron was regarded as the $\mathrm{sp}_{2}$-carbon, and the bond length and the bond angle were assumed to be $1.50 \AA$ and $120.0^{\circ}$, respectively. The conformation of a short sequence consisting of the radical end unit and a penultimate monomer unit was taken into account, and conformation energies were calculated by varying the parameters of internal rotation angles $\left(\tau_{1}, \tau_{2}, \tau_{3}, \tau_{4}\right.$, and $\left.\tau_{5}\right)$. (Figure 1). The calculations were made at $5^{\circ}$ intervals of $\tau_{2}$ and at $30^{\circ}$ intervals of $\tau_{1}, \tau_{3}, \tau_{4}$, and $\tau_{5} . \tau_{1}, \tau_{2}, \tau_{3}, \tau_{4}$, and $\tau_{5}=180^{\circ}$ defines the planar zigzag conformation as drawn in Figure 1. A computer program developed by Tadokoro et al. ${ }^{9}$ was used for these calculations.

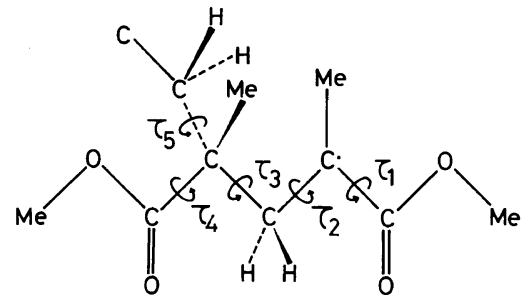

Figure 1. Schematic diagram of poly(MMA) radical. $\tau_{1}, \tau_{2}, \tau_{3}, \tau_{4}$, and $\tau_{5}=180^{\circ}$ defines the planar zigzag conformation.

A spectral simulation for distributed conformation was performed by Iwasaki's method ${ }^{10}$ and simulations for the overlap of two spectra of two conformations were carried out by a JEOL ESR simulation program 0398.

\section{RESULTS AND DISCUSSION}

The ESR spectrum of the polymerization system of TPMA in MTHF was measured in the temperature range from $-90^{\circ} \mathrm{C}$ to $30^{\circ} \mathrm{C}$. The results are shown in Figure 2. No signal was observed in the absence of TPMA under the same conditions. The 9 $(5+4)$-line spectrum with an equal spacing of about 11.5 gauss was observed at $-90^{\circ} \mathrm{C}$, and was consistent with the usual $9(5+4)$-line spectrum of the poly(MMA) radical in aromatic glassy medium except for the intensity distribution. ${ }^{5}$ Since the poly(TPMA) radical is a homolog of the poly(MMA) radical, we may conclude that the ESR spectrum is definitely the spectrum of the propagating radical of TPMA. The ESR spectrum changed with the measurement temperature. The higher the temperature, the weaker were the 4 lines in the 9 $(5+4)$-line spectrum. These lines almost disappeared at $-50^{\circ} \mathrm{C}$. As the polymerization temperature was raised, the 5-line spectrum at $-50^{\circ} \mathrm{C}$ changed into a $13(5+8)$-line spectrum at $0^{\circ} \mathrm{C}$. The 8 lines became weaker and broader with increase in temperature. At $30^{\circ} \mathrm{C}$, a 5 -line spectrum with an equal spacing of 23 gauss was observed again. The yield of polymer obtained after measurement at $30^{\circ} \mathrm{C}$ was less than $5 \%$. When the temperature was lowered from $30^{\circ} \mathrm{C}$ to $-90^{\circ} \mathrm{C}$, similar spectra were observed at each temperature, although their signals became broader as the temperature was lowered.

Variation in the ESR spectrum with the polymerization temperature may perhaps be correlated with 

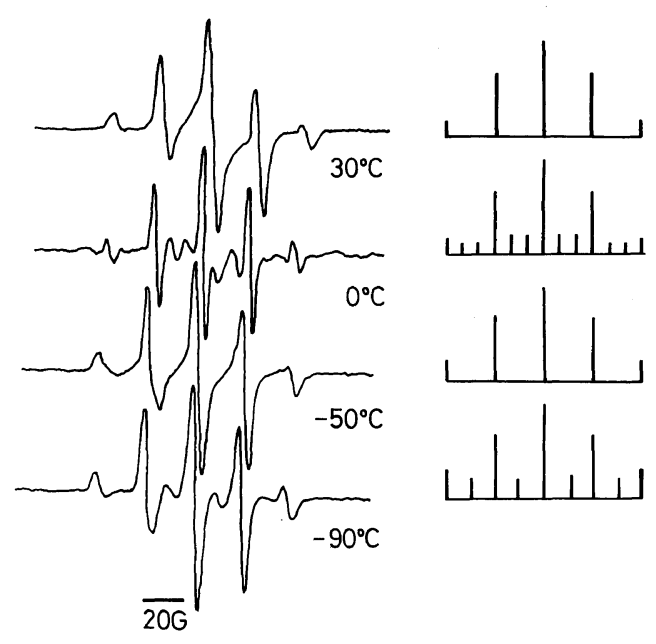

Figure 2. Temperature dependence of the ESR spectrum of poly(TPMA) radical obtained in MTHF. $[\mathrm{TPMA}]=2 \mathrm{M}$ and $[\mathrm{DTBP}]=0.1 \mathrm{M}$.

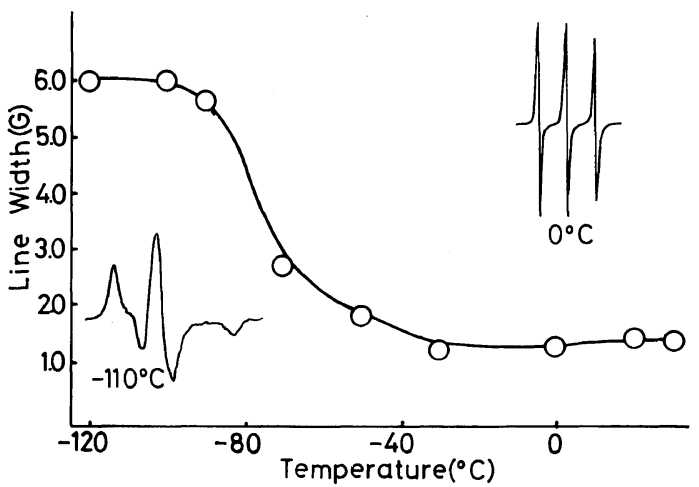

Figure 3. Variation in the line width of the center line of Tempol with temperature in a mixture of TPMA (2 M) and MTHF. [Tempol] $=10^{-4} \mathrm{M}$.

the change in the state of the medium. In order to obtain information on the thermal behavior of the medium, the temperature dependence of the line width of central line in the ESR spectrum of 2,2,6,6tetramethylpiperidine-1-oxyl (Tempol) added to the system at a concentration of $10^{-4} \mathrm{M}$ was studied. ${ }^{11}$ The results are shown in Figure 3. The ESR spectra showed an amorphous pattern below $-80^{\circ} \mathrm{C}{ }^{12}$ This remarkable change was observed within a temperature region from $-85^{\circ} \mathrm{C}$ to $-50^{\circ} \mathrm{C}$, suggesting that the polymerization system was in a glassy state below $-80^{\circ} \mathrm{C}$, and in a fluid state above $-50^{\circ} \mathrm{C}$. This indicates that the addition of TPMA to MTHF ( $2 \mathrm{M})$ brings about a rise in the upper
A

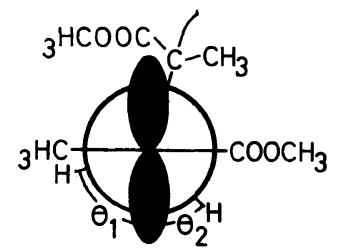

B

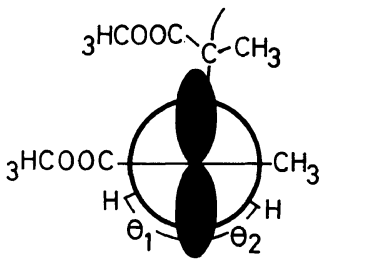

Figure 4. Two stable conformations of poly(MMA) radical.

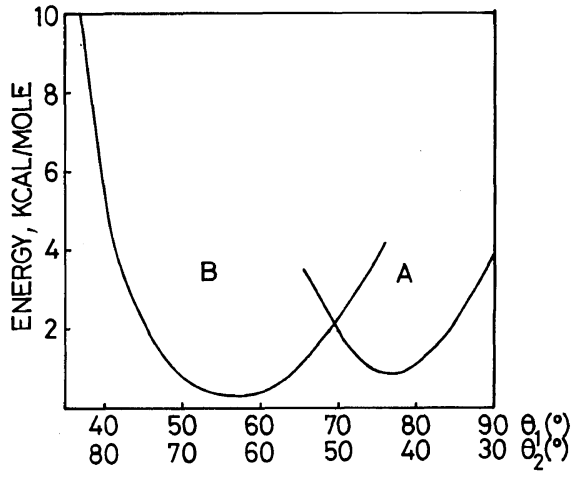

Figure 5. Conformation energies of poly(MMA) radical as function of the dihedral angle of $\beta$-protons. A and $B$ interchange through free rotation of radical end.

limits of the temperature of the glassy state, since MTHF or MTHF-MMA (2M) is in a fluid state above $-130^{\circ} \mathrm{C}$. Thus, the change in the ESR spectrum with rising temperature, from $-90^{\circ} \mathrm{C}$ to $-50^{\circ} \mathrm{C}$, seems to be correlated with the mobility of the propagating radical.

The following three concepts have been independently proposed for elucidating the spectrum of the poly(MMA) radical: 1) Gaussian distribution about the most stable conformation, ${ }^{10,13}$ 2) exchange of two protons due to hindered oscillation, ${ }^{14}$ and 3) two conformations. ${ }^{15-18}$

Whether or not there are two stable conformations in the radical end still remains theoretically unsolved. Since poly(methacrylate) radicals have the same spectra but different intensity distributions, they are considered to be similar in confor- 
mation. In order to understand the conformation of the poly(methacrylate) radical end, calculations of the conformation energy of the poly(MMA) radical were carried out, taking into consideration the conformations of the last two monomer units.

The calculation results indicate that the propagating radical has two stable conformations (conformation $\mathrm{A}, \tau_{1}=300^{\circ}, \tau_{2}=75^{\circ}, \tau_{3}=60^{\circ}, \tau_{4}=0^{\circ}$, and $\tau_{5}=300^{\circ}$, and conformation $\mathrm{B}, \tau_{1}=300^{\circ}, \tau_{2}=265^{\circ}$, $\tau_{3}=60^{\circ}, \tau_{4}=0^{\circ}$, and $\left.\tau_{5}=300^{\circ}\right)$. Dihedral angles $\left(\theta_{1}\right.$ and $\theta_{2}$ ) of $\beta$-protons with the $\pi$-orbital of the unpaired electron were calculated from the value of $\phi$ for the two stable conformations. (Figure 4). Changes in the conformation energy with free rotation in conformations $\mathrm{A}$ and $\mathrm{B}$ are shown in Figure 5. The dihedral angles (conformation $\mathrm{A}$, $\theta_{1}=75^{\circ}$ and $\theta_{2}=45^{\circ}$, and conformation $\mathrm{B}, \theta_{1}=65^{\circ}$ and $\theta_{2}=55^{\circ}$ ) were found to be consistent with the view that the 9-line spectrum of the poly(MMA) radical in rigid glass is an overlap of the spectra of the two conformations (conformation $\mathrm{A}$ and conformation $\left.\mathbf{B}^{\prime}, \theta_{1}=\theta_{2}=60^{\circ}\right) .{ }^{6,15-18}$ Some difference in the dihedral angles between the energy calculation and the experimental results is due possibly to the fact that intermolecular interaction with solvent molecules ${ }^{3}$ was not taken into account in the energy calculation. Since weak interaction between the propagating radical and solvent is more likely in the glassy state, where the mobility of the propagating radical remarkably decreases, than that in liquid state, the conformation in the glassy state may be somewhat different from that in the liquid state.

The ESR spectrum of the poly(TPMA) radical at $-90^{\circ} \mathrm{C}$ was the same as that of the poly(MMA) radical in the glassy aromatic medium at $-120^{\circ} \mathrm{C}$ but the intensity distribution was different. Thus, the 9-line spectrum of the poly(TPMA) radical may also be explained on the basis of the two conformations, and the spectrum was simulated as overlap of spectra of two conformations (conformation A: conformation $\mathrm{B}^{\prime}=9: 1$ ) as shown in Figure 6. The ratio of $A$ to $B$ in poly(TPMA) radical was different from that in poly(MMA) radical obtained in frozen anisole $(6: 4) .{ }^{19}$ The difference might be ascribed to the bulkiness of ester group, because conformation B increased with decreasing the bulkiness of the group. ${ }^{19}$ The 13-line spectrum was observed at $0^{\circ} \mathrm{C}$. (Figure 2). The intensity distribution of the spectrum cannot be explained by a single conformation $\left(\theta_{1}=65^{\circ}\right.$ and $\left.\theta_{2}=55^{\circ}\right)$ which leads to a 13-line

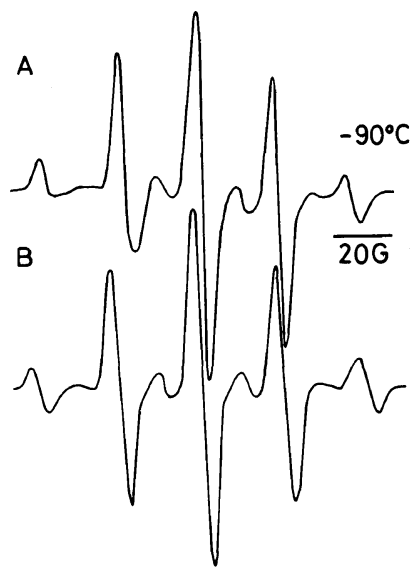

Figure 6. ESR spectra of poly(TPMA) radical, assuming an overlap of the two conformations. (A) Observed ESR spectrum. (B) Simulated ESR spectrum, conformation $\mathrm{A}$ : conformation $\mathrm{B}=9: 1$.

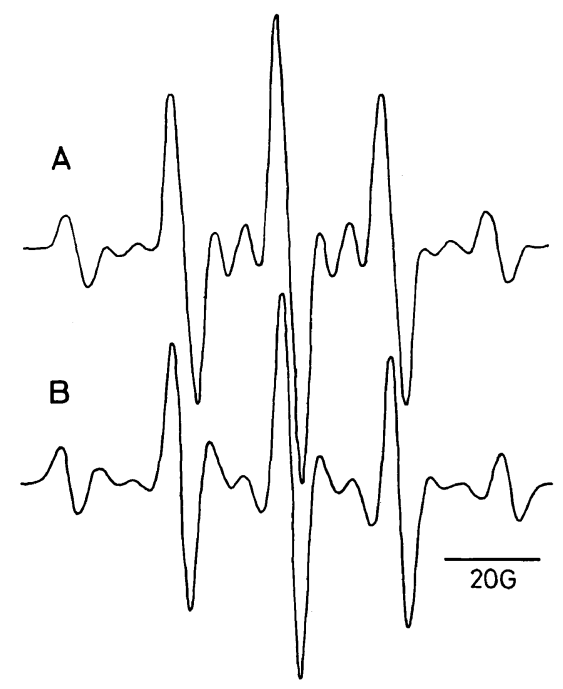

Figure 7. Simulated ESR spectra of the poly(TPMA) radical, assuming an overlap of the two conformations (A) and a Gaussian distribution about conformation (B). (A) Conformation $\mathrm{A}$ :conformation $\mathrm{B}=8: 3$, line width $=4$ gauss. (B) Half-height width $=5^{\circ}$, line width $=$ 3 gauss.

spectrum $(1: 1: 1: 4: 3: 3: 6: 3: 3: 4: 1: 1: 1)$. The 13-line spectrum was simulated either by an overlap of the two conformations (conformation A: conformation $\mathrm{B}=8: 3$ ) or by the Gaussian distribution about the most stable conformation $\left(\theta_{1}=65^{\circ}\right.$ and $\theta_{2}=55^{\circ}$ ) where the half-height width was assumed to be $5^{\circ}$. (Figure 7). In order to decide the actual 


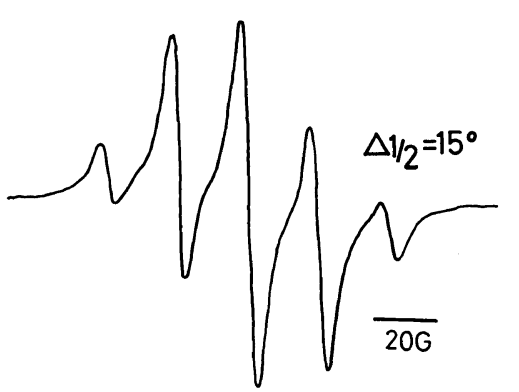

Figure 8. Simulated ESR spectrum of poly(TPMA) radical assuming a Gaussian distribution about conformation B. Half-height width $=15^{\circ}$, line width $=6$ gauss.

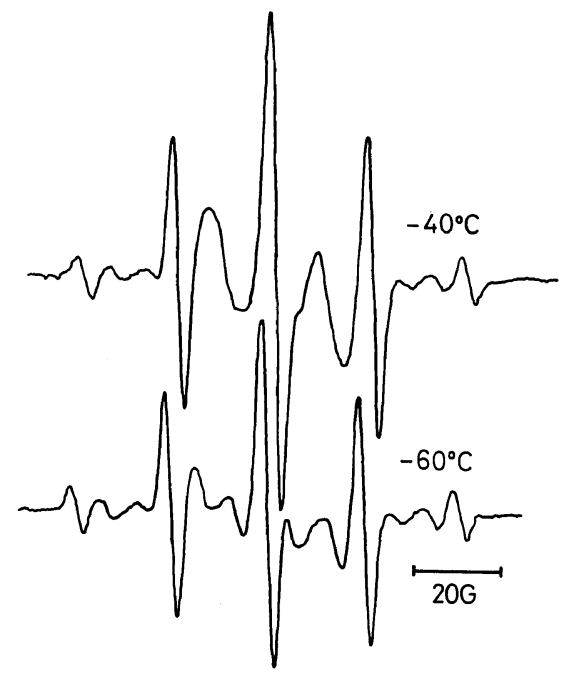

Figure 9. ESR spectra of poly $(\alpha$-methylbenzyl methacrylate) radical at $-40^{\circ} \mathrm{C}$ and $-60^{\circ} \mathrm{C}$.

cause of simulation, the temperature dependence of the spectrum was considered on the basis of these two explanations. First, let us consider the spectrum change in relation to the Gaussian distribution about the most stable conformation (conformation B). ${ }^{10,13}$ The 5-line spectra at $30^{\circ} \mathrm{C}$ and $-50^{\circ} \mathrm{C}$ were simulated by a distribution whose half-height width was taken to be $15^{\circ}$ (Figure 8) since both spectra are similar. This indicates the physically meaningless phenomenon that the distribution becomes wider at temperatures both higher and lower than that at $0^{\circ} \mathrm{C}$. This explanation is thus ruled out. Next, let us consider the spectrum change with regard to the two conformations. Interchange between the two stable conformations (A and B) is possible in a liquid, since the radical end rotates at a rate dependent on

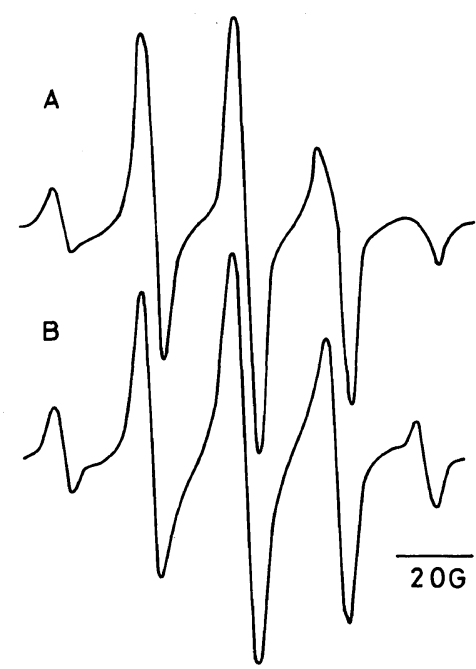

Figure 10. Simulated ESR spectrum of the poly(TPMA) radical, assuming a Gaussian distribution about the two conformations. (A) Observed ESR spectrum. (B) Simulated ESR spectrum, assuming a Gaussian distribution about two conformations. Half-height width $=10^{\circ}$, line width $=4$ gauss.

temperature. The 13 -line spectrum at $0^{\circ} \mathrm{C}$ shows that the average lifetime of exchange due to oscillation is small enough compared with difference in the resonance frequencies of the $\beta$-protons. ${ }^{20}$ The higher the observation temperature, the greater the frequency of oscillation. When the average lifetime becomes short, a greater extent of exchange may be observed in the spectrum. ${ }^{20}$ Change in the spectrum from $0^{\circ} \mathrm{C}$ to $30^{\circ} \mathrm{C}$ shows that 8 inner lines disappeared with a greater degree of exchange. The coalescence of the peaks can probably be observed at time-average resonant-field positions at higher temperature. However, the propagating radical was too unstable to allow detection of the spectrum at a temperature higher than $30^{\circ} \mathrm{C}$. The coalescence peaks were observed in the poly $(\alpha$-methylbenzyl methacrylate) radical, which has a much small ester group than the poly(TPMA) radical, in a lower temperature range from $-60^{\circ} \mathrm{C}$ to $-40^{\circ} \mathrm{C}$, where it is stable. (Figure 9). When the temperature was made $-60^{\circ} \mathrm{C}$ again, the spectrum went back to the original one. Therefore, the coalescence of the peaks shows that interchange between the two stable conformations occurs.

The temperature dependence of the spectrum pattern from $-90^{\circ} \mathrm{C}$ to $-50^{\circ}$ cannot be explained 
Table I. Assignments of poly(TPMA) radical spectra

\begin{tabular}{ccl}
\hline \multicolumn{1}{c}{ Temperature } & Spectrum & \multicolumn{1}{c}{ Assignment } \\
\hline${ }^{\circ} \mathrm{C}$ & 5 lines & $\begin{array}{l}\text { Exchange } \\
\text { broadening } \\
\text { between A and B } \\
\text { Overlap of A and B } \\
(8: 3)\end{array}$ \\
0 & 13 lines & $\begin{array}{l}\text { Distribution (Slow } \\
\text { interchange) } \\
\text { Overlap of A and B' } \\
(9: 1)\end{array}$ \\
\hline-50 & 9 lines
\end{tabular}

on the basis of exchange broadening, since it is unreasonable for exchange broadening to occur twice in the system. Another explanation must be found for this phenomenon. The change in the spectrum pattern can be explained as a result of the slow rotation of the radical due to the high viscosity of the system and the bulkiness of the side group of the polymer. Since the results shown in Figure 3 indicate no rapid interchange between the two conformations below $-50^{\circ} \mathrm{C}$, multi-conformations in the intermediate region between $\mathrm{A}$ and $\mathrm{B}$ must be considered. However, an accurate potential curve about the two stable conformations is not available at the present. Consequently, a 5-line spectrum at $-50^{\circ} \mathrm{C}$ was simulated tentatively on the assumption that the distribution about the two stable conformations was Gaussian and that the ratio of conformation $\mathrm{A}$ to $\mathrm{B}$ was $9: 1$. The spectrum was fully developed with a half-height width of $10^{\circ}$ (Figure 10).

Thus, the temperature dependence of the ESR spectrum of the poly(TPMA) radical is reasonably explained in terms of the concept of the two conformations in which the interchange between them by means of free rotation of the radical end is taken into account. The two conformations in free radicals have been reported for a series of nitroxides $\mathrm{RCH}_{2} \mathrm{~N}\left(\mathrm{Bu}^{t}\right) \mathrm{O}$ - in which the group $\mathrm{R}$ has a chiral center. ${ }^{21}$ Assignments of the spectra of the poly(TPMA) radical are summarized in Table I.

The concept we have proposed seems an adequate explanation for the temperature dependence of ESR spectra of many poly(methacrylate)s radicals. The ESR study on other poly(methacrylate) radicals is now in progress.

Acknowledgement. We should like to thank Professor H. Tadokoro for allowing us to use the computer program in making calculations of conformation energy. We are also indebted to Professors K. Kuwata and Y. Chatani, and Mr. H. Kusuyama for their kind assistance in carrying out the computer calculations and their valuable comments. This research was supported in part by a Grant-in-Aid for Scientific Research from the Ministry of Education, Science and Culture of Japan.

\section{REFERENCES}

1. M. Kamachi, J. Satoh, D. J. Liaw, and S. Nozakura, Macromolecules, 10, 501 (1977).

2. M. Kamachi, D. J. Liaw, and S. Nozakura, Polym. J., 9, 307 (1977).

3. M. Kamachi, J. Satoh, and S. Nozakura, J. Polym. Sci., Polym. Chem. Ed., 16, 1789 (1978).

4. M. Kamachi, D. J. Liaw, and S. Nozakura, Polym. J., 11, 921 (1979).

5. M. Kamachi, D. J. Liaw, and S. Nozakura, Polym. J., 13, 41 (1981).

6. M. Kamachi, M. Kohno, D. J. Liaw, and S. Katsuki, Polym. J., 10, 69 (1978).

7. H. Yuki, K. Hatada, T. Niinomi, and Y. Kikuchi, Polym. J., 1, 36 (1970).

8. H. Tadokoro, "Structure of Crystalline Polymers," Wiley, New York, 1979, pp 323-352.

9. H. Tadokoro, K. Tai, M. Yokoyama, and M. Kobayashi, J. Polym. Sci., Polym. Phys. Ed., 11, 825 (1973).

10. M. Iwasaki and Y. Sakai, J. Polym. Sci., A-1, 7, 1537 (1969).

11. J. H. Freed, "Spin Labeling, Theory and Applications," L. J. Berliner, Ed., New York; 1976, pp 53-121.

12. E. Murakami and J. Sohma, Jpn. J. Appl. Phys., 18, 415 (1979).

13. M. H. Bowden and J. H. O'Donnell, J. Phys. Chem., 72, 1577 (1968).

14. Y. Sakai and M. Iwasaki, J. Polym. Sci., A-1, 7, 1749 (1969).

15. J. Sohma, T. Komatsu, and H. Kashiwabara, J. Polym. Sci., B, 3, 287 (1965).

16. M. S. Symmons, J. Chem. Soc., 1186 (1963).

17. S. E. Bresler, E. N. Kozbekov, V. N. Fomichev, and V. V. Shadin, Makromol. Chem., 175, 2875 (1973).

18. J. A. Haris, O. Hinojosa, and J. C. Arther, Jr., J. Polym. Sci., Polym. Chem. Ed., 11, 3215 (1973).

19. M. Kamachi, Y. Kuwae, and S. Nozakura, to be published. 


\section{Conformation of the Propagating Radical}

20. J. E. Wertz and J. R. Bolton, "Electron Spin Resonance, Elementary Theory and Practical Applications," McGraw-Hill, New York, 1972, pp
$192-222$.

21. B. C. Gilbert and M. Trenwith, J. Chem. Soc., Perkin Trans 2, 1834 (1973). 